\title{
Leitthema
}

Orthopäde 2014 • 43:1089-1095

DOI 10.1007/s00132-014-3051-2

Online publiziert: 21. November 2014

(c) Die Autor(en) 2014. Dieser Artikel ist auf

Springerlink.com mit Open Access verfügbar
J. Schröter ${ }^{1}$ M. Lechterbeck ${ }^{2}$. F. Hartmann ${ }^{2}$ - E. Gercek ${ }^{2}$

${ }^{1}$ Orthopädie, Klaus-Miehlke-Klinik, Wiesbaden, Deutschland

2 Zentrum für Unfallchirurgie und Orthopädie, Gemeinschaftsklinikum Mittelrhein - Ev. Stift St. Martin, Koblenz, Deutschland

\section{Strukturierte Rehabilitation nach lumbaler Wirbelsäulenoperation}

\section{Subakute Behandlungsphase}

\begin{abstract}
Bislang gibt es nach Wirbelsäulenoperationen keine klare Handlungsempfehlung für die postoperative Rehabilitation und den Belastungsaufbau. Lediglich in einigen mittlerweile abgelaufenen AWMF-Leitlinien (Arbeitsgemeinschaft Medizinisch Wissenschaftlicher Fachgesellschaften), z. B. nach Bandscheibenoperation in der S1-Leitlinie der Deutschen Gesellschaft für Orthopädie und Orthopädische Chirurgie (DGOOC) von 2005 „Rehabilitation bei Bandscheibenvorfall mit radikulärer Symptomatik" werden tägliche krankengymnastische Übungen unter Stabilisation des operierten Bewegungssegments empfohlen.
\end{abstract}

In diese genannten Übungen eingebunden ist ein isometrisches und auch ein aufbauendes Muskeltraining, befundabhängig die Kräftigung der Rückenstreckmuskulatur durch medizinische Trainingstherapie und andere bewährte Stabilisierungsverfahren. Zur Therapiedichte werden ca. fünf Leistungen pro Tag empfohlen, davon zwei bis drei aktive und zwei passive Leistungen, die aus den Bereichen Einzelkrankengymnastik, Gruppenkrankengymnastik im Bewegungsbad, Sequenztraining (medizinische Trainingstherapie) und Sporttherapie kommen sollen.

Weder der Zeitpunkt des Beginns und die Dauer einer gezielten Rehabilitation, noch die Dauer einer postoperativen körperlichen Schonung oder die Möglichkeit einer direkten postoperativen Anschlussheilbehandlung nach Wirbelsäulenopera- tionen, ist festgelegt. Exakte Empfehlungen in der Literatur finden sich nicht.

Bedingt durch die Optimierung der Operationstechniken mit Einführung von sofort belastungsstabilen Spondylodeseverfahren ergeben sich auch für die direkte postoperative Rehabilitation neue Möglichkeiten. Im Rahmen dieser prospektiven Multicenterstudie sollte daher evaluiert werden, wie der Effekt einer nach 2 Wochen postoperativ begonnenen Rehabilitation (ambulante Rehabilitation oder Anschlussheilbehandlung) auf die gesundheitsbezogene Lebensqualität und Beeinträchtigung durch Rückenschmerzen bei diesen Patienten ist. Bewusst wurde die Akutbehandlungsphase mit komplikationsloser Wundheilung (in der Regel bis 14 Tage) ausgespart. Die Akutbehandlungsphase bildet den initialen kurativen Prozess ab, der den morphologischen Prozess der Gewebeheilung beschreibt.

Der gewählte Beobachtungszeitraum ist somit die subakute Behandlungsphase nach Abschluss der Wundheilung ca. 2 Wochen postoperativ. Konsens war, dass die Intensität und die Form der angewandten Therapien in dieser Phase gesteigert werden muss.

\section{Material und Methoden}

Unter der Kenntnis, dass ein Rehabilitationsteam definierte Rehabilitationskriterien und Standards benötigt, um den Rehabilitationsprozess und die Therapieintensität festzulegen, wurden drei Belastungsgruppen definiert [15]. Gruppe A umfasst die Patienten mit ei- ner gegebenen Primärstabilität ohne wesentliche Einschränkung hinsichtlich des postoperativen Belastungsaufbaus, Gruppe $B$ umfasst Patienten mit geringen Einschränkungen und Gruppe $\mathrm{C}$ betrifft $\mathrm{Pa}-$ tienten, welche eine geringere Primärstabilität aufweisen. Aus der intensiven Diskussion der teilnehmenden Institutionen ergab sich schlussendlich die Erkenntnis, dass eine alleinige Einteilung der Patienten hinsichtlich der Ausgangsdiagnose bezüglich der zu wählenden postoperativen Belastungsfähigkeit nicht geeignet ist. Es wurde daher im Konsens vereinbart, dass ein Patient jeweils durch seinen Operateur in eine der drei unterschiedlichen Belastungsgruppen eingeteilt werden sollte.

\section{》) Der Patient wird durch seinen Operateur in eine Belastungsgruppe eingeteilt}

Im Rahmen einer umfangreichen Diskussion der teilnehmenden Institutionen und Berufsgruppen über etablierte rehabilitative konservative Therapieverfahren erfolgte eine Festlegung der zu wählenden Therapieformen im Bereich der Physiotherapie und physikalischen Therapie aufgeteilt nach den Gruppen und nach dem Zeitpunkt in der subakuten Behandlungsphase entsprechend der in $\bullet$ Tab. 1 angegebenen Inhalte.

Die vorliegende Publikation basiert u. a. auf Daten aus der Dissertation von M. Lechterbeck, Universität Mainz. 


\begin{tabular}{|c|c|c|c|}
\hline & Gruppe C - starke Einschränkung & Gruppe B - geringe Einschränkung & Gruppe A - kaum Einschränkung \\
\hline $\begin{array}{l}\text { 3. Woche } \\
\text { postoperativ }\end{array}$ & $\begin{array}{l}\text { Einzelgymnastik: } \\
\text { Grundspannungsübungen, } \\
\text { Neurodynamik, } \\
\text { Brunkow, } \\
\text { Transfers (ADL), } \\
\text { isometrisches Krafttraining, } \\
\text { Haltungskorrektur } \\
\text { (Aufrichtung, leichte BWS Mobilistation), } \\
\text { Dehnung passiv (z. B.: ischiocrurale Mm.). } \\
\text { Bewegungsbad: } \\
\text { ohne Trainingsgeräte, } \\
\text { stabilisierende Übungen, Lauf ABC, } \\
\text { keine aktive Mobilisation der WS. } \\
\text { Hallengruppe: } \\
\text { Rückenschule }\end{array}$ & $\begin{array}{l}\text { Wie Gruppe } 3 \text { plus: } \\
\text { Einzelgymnastik: } \\
\text { schmerzfreie Mobilisation LWS in alle } \\
\text { Richtungen, } \\
\text { PNF, } \\
\text { aktive Dehnung, ischiocrurale Mm., } \\
\text { Hüftbeuger in RL, Adduktoren, Gastrocne- } \\
\text { mius, Quadriceps, Nervenmobilisation, } \\
\text { leichtes Stabilisierungstraining } \\
\text { (z. B. Einbeinstand), } \\
\text { Koordinationstraining. } \\
\text { Bewegungsbad: } \\
\text { leichte Widerstände, } \\
\text { schmerzfreie Rotation. } \\
\text { MTT: } \\
\text { leichte Widerstände, Seilzug (keine Rot.), } \\
\text { Butterfly, Lat-Zug, Sitzfahrrad, ggf. Ergo- } \\
\text { meter }\end{array}$ & $\begin{array}{l}\text { Wie Gruppe } 3 \text { und } 2 \text { plus: } \\
\text { Einzelgymnastik: } \\
\text { Bauchmuskeltraining dynamisch, } \\
\text { Bridging, } \\
\text { Einbeinstand, } \\
\text { Stabilisation mit Flexibar, Aerostep, Mini- } \\
\text { trampolin. } \\
\text { Bewegungsbad: } \\
\text { alle Bewegungen im schmerzfreien Bereich. } \\
\text { Hallengruppe: } \\
\text { Übungen in verschiedenen Ausgangspositio- } \\
\text { nen ohne größere Einschränkungen. } \\
\text { MTT: } \\
\text { (höhere) angepasste Widerstände, Butterfly, } \\
\text { Hüfttrainer, Seilzug mit Aerostep oder Thera- } \\
\text { piekreisel, Ergometer }\end{array}$ \\
\hline $\begin{array}{l}\text { 4. Woche } \\
\text { postoperativ }\end{array}$ & $\begin{array}{l}\text { Hubfreie Mobilisation (Becken kippen), } \\
\text { Beginn dynamischer Kräftigung } \\
\text { (z. B.: leichte Kniebeuge) }\end{array}$ & $\begin{array}{l}\text { Kräftigung mit Theraband, Flexibar, } \\
\text { Dehnung Mm. tensor f.l., Piriformis, } \\
\text { Hüftbeuger im Stand. } \\
\text { Hallengruppe: } \\
\text { Übungen im Schmerzfreien Bereich in ver- } \\
\text { schiedenen Ausgangspositionen. } \\
\text { Sitzen/Liegen/Stehen } \\
\text { (keine forcierte Rotation) }\end{array}$ & $\begin{array}{l}\text { Kräftigungsübungen mit Zusatzlasten } \\
\text { (Hantel/Langhantel) }\end{array}$ \\
\hline $\begin{array}{l}\text { 5. Woche } \\
\text { postoperativ }\end{array}$ & $\begin{array}{l}\text { Bridging, leichte Rotation, } \\
\text { Bücken, Heben (geringe Last). } \\
\text { MTT: } \\
\text { Übungen am Seilzug mit geringer Last } \\
\text { (ohne Rotation der WS) }\end{array}$ & Weitere langsame Steigerung & Walking \\
\hline
\end{tabular}

Zur Evaluation wurden die etablierten Validierungsinstrumente „Oswestry Disability Score“ (ODI), „Short Form 12 Health Survey" (SF-12) und die visuelle Analogskala (VAS) sowohl zu Anfang der Rehabilitation, als auch zum Ende der Rehabilitation erhoben.

Die Untersuchung fand von April 2013 bis Dezember 2013 statt. Ein positives Votum der Ethikkommission des Landes Rheinland-Pfalz liegt vor. Für diese Studie wurden 124 Patienten aus 7 Akutkliniken gewonnen, die sich einer Operation an der LWS unterziehen mussten, die Akutbehandlungsphase abgeschlossen hatten und einer stationären oder intensivierten ambulanten Rehabilitation zugeführt wurden. Bei 96,8\% wurde stationär in 2 Rehabilitationskliniken eine Anschlussheilbehandlung durchgeführt, 3,2\% unterzogen sich einer ganztätig ambulanten Anschlussheilbehandlung in 3 Rehabilitationszentren. Die Rehabilitanden waren durchschnittlich 61,5 Jah- re alt, (45,9\% waren $\leq 65$ Jahre alt, 54,1\% waren $>65$ Jahren alt) der Frauenanteil betrug $63,7 \%(n=79)$.

Neben Spondylodesen bei angeborenen und erworbenen Erkrankungen, längerstreckigen Fusionsoperationen unabhängig von der Zugangswahl (ventral, dorsal oder kombiniert dorsoventral) wurden auch Patienten nach Bandscheibenoperationen mit eingeschlossen. Die Verteilung war wie folgt: 32 Spondylodesen, 10 langstreckige Fusionen, 60 Operationen nach Bandscheibenvorfällen sowie 22 andere Operationen (Verteilung und Zuordnung zu den Gruppen s. • Tab. 2).

Die Dauer der Anschlussheilbehandlung betrug im Mittel 20,5 Tage mit einer Standardabweichung von 2,7 Tagen.

\section{Messinstrumente}

Um den Zustand der Patienten abbilden zu können, wurden psychische und somatische Parameter mittels etablier- ter Assessmentinstrumente erfasst. Zum Erhebungszeitpunkt t1 (Reha-Aufnahme) wurden die VAS, der ODI und der SF-12 eingesetzt. Dieses Vorgehen wurde bei der Entlassung aus der Rehabilitation wiederholt. Aufgrund der umfangreicheren Bestimmung der faktoriellen Parameter der Messinstrumente wurden die Ergebnisse zum Zeitpunkt der Entlassung aus der Rehabilitation (t2) untersucht.

\section{„Oswestry Disability Index"}

Der ODI erfasst die Beeinträchtigungen durch Rückenschmerzen mit 10 Items. Neben der Schmerzstärke werden Bereiche des alltäglichen Lebens, wie Körperpflege, Gehen, Schlafen und Sozialleben erfasst [5]. Zur Reliabilität und Validität gibt es viele internationale Untersuchungen, die die Aussagekraft des ODI beweisen [2]. Die Fragen wurden jeweils anhand einer 6-stufigen Skala $(0=$ keine Beeinträchtigung, $5=$ Unfähigkeit, eine 
Orthopäde 2014·43:1089-1095 DOI 10.1007/s00132-014-3051-2

○ Die Autor(en) 2014. Dieser Artikel ist auf Springerlink.com mit Open Access verfügbar

\section{J. Schröter · M. Lechterbeck · F. Hartmann · E. Gercek}

\section{Strukturierte Rehabilitation nach lumbaler Wirbelsäulenoperation. Subakute Behandlungsphase}

\section{Zusammenfassung}

Hintergrund. Bislang gibt es keine einheitlichen Empfehlungen zur Rehabilitation von Patienten, bei denen eine Operation im Bereich der Lendenwirbelsäule (LWS) durchgeführt wurde. Wenngleich sich die Operationsmethoden im Laufe der letzten Jahre deutlich verbessert haben und somit die Belastungsfähigkeit nach den Operationen erhöht ist, erfolgte bislang keine Anpassung der konservativen rehabilitativen Nachbehandlung. Ein früher Start einer strukturierten Rehabilitation nach der Akutbehandlungsphase wird von Operateuren oft noch skeptisch betrachtet.

Untersuchung. Um den Effekt einer strukturierten Rehabilitation nach Operationen im LWS-Bereich in der subakuten Behandlungsphase (beginnend in der 3 . Woche nach der Operation) zu untersuchen, erfolgte ein Zu- sammenschluss von sieben wirbelsäulenoperierenden Kliniken, zwei stationären und drei ambulanten Rehabilitationszentren im RheinMain-Gebiet. Bei dieser prospektiven Untersuchung des Rehabilitationsverlaufes wurden 124 Patienten durch ihren Operateur in eine definierte Belastungsgruppe $(A / B / C)$, unabhängig von Diagnose und Operationsverfahren eingeteilt. Für jede Gruppe wurden vorab von allen Beteiligten die Therapieinhalte festgelegt.

Ergebnisse. Mit den Validierungsinstrumenten visuelle Analogskala (VAS), "Oswestry Disability Score" (ODI) und "short form 12" (SF-12) wurden die Veränderungen der gesundheitsbezogene Lebensqualität und von Beeinträchtigungen durch Rückenschmerzen in den Gruppen evaluiert. In allen drei Gruppen konnte eine deutliche, meist signifikante
Verbesserung in ODI, VAS und SF-12 gezeigt werden. Reoperationswürdige Komplikationen traten nicht auf.

Zusammenfassung. Es konnte festgestellt werden, dass durch eine direkt an die Operation anschließende strukturierte Rehabilitation, welche vom Operateur indikationsunabhängig auf die Belastungsfähigkeit des Patienten abgestimmt ist, eine deutliche Verbesserung in den Parametern Schmerz und Lebensqualität erzielt werden kann, ohne das wesentliche Komplikationen auftreten.

\section{Schlüsselwörter}

Wirbelsäulenchirurgie · Lendenwirbelsäule . Akutbehandlungsphase - Lebensqualität · Spondylodese

\section{Structured rehabilitation after lumbar spine surgery. Subacute treatment phase}

\section{Abstract}

Background. There are currently no uniform standards regarding rehabilitation of patients after lumbar spine surgery. Due to significant improvements in surgical methods in recent years, an increase in postoperative training intensity is now possible. Conservative rehabilitation has yet to adapt to this reality. Earlier initiation of structured rehabilitation after the acute phase is often regarded with skepticism.

Objective. To evaluate the effect of structured rehabilitation after lumbar spine surgery in the early phase of treatment ( 2 weeks after surgery), a group of seven spinal surgery clinics, two inpatient and three outpatient re- habilitation centers in the Rhine-Main area in Germany was formed.

Materials and methods. In this prospective study, 124 patients were divided into groups (A/B/C) by their surgeon, regardless of diagnosis and surgical procedure. For each group of participants, the content of therapy was preplanned.

Results. The statistical analysis using the visual analog scale (VAS), Oswestry Disability Index (ODI), and short form-12 health survey (SF-12) to evaluate changes in impairment caused by back pain and in health-related quality of life was evaluated. In all three groups, significant improvements in VAS, ODI and SF-12 were shown. Re-operation was unnecessary due to the absence of postoperative complications.

Conclusion. A structured postoperative rehabilitation program results in significant improvements in the parameters of pain and quality of life, and does not increase the risk of postoperative complications.

\section{Keywords}

Spine surgery - Lumbar spine . Acute treatment phase - Quality of life . Spondylosyndesis
Aktivität auszuführen bzw. sehr starke Schmerzen bei der Ausführung) gestellt. Die Auswertung erfolgte entsprechend der Angaben von Fairbank u. Pynsent [1]. Die resultierenden Prozentangaben stellen dabei den Anteil der Beeinträchtigung im alltäglichen Leben dar $(0=$ keine Beeinträchtigung, 1,0= maximale Beeinträchtigung).

\section{"Short Form-12 Health Survey"}

Die gesundheitsbezogene Lebensqualität wurde mit dem SF-12 evaluiert [11]. Dieser erfasst 12 Items mit den Dimensionen
„Körperliche Gesundheit“ (SF-12 KG: 6 Items) und „Psychische Gesundheit“ (SF-12 PG: 6 Items). Die Auswertung des SF-12 erfolgt durch eine Codierung und eine Gewichtung der Variablen [11]. Hohe Skalenwerte zeigen dabei eine hohe körperliche bzw. psychische Lebensqualität. Die guten psychometrischen Eigenschaften konnten in der Literatur belegt werden $[7,8,13]$.

\section{Visuelle Analogskala}

Die VAS ist eine Skala zur Messung v. a. subjektiver Empfindungen. Es handelt sich um eine Linie, deren Endpunkte extreme Zustände darstellen, „kein Schmerz“ und „unerträglicher Schmerz“. Der Befragte markiert seine subjektive Empfindung durch einen vertikalen Strich auf der Linie. Der angegebene Wert wird anschließend mit einer definierten Skala, von 0-10 quantifiziert. Der Patient sieht die Zahlen dabei nicht $[3,4]$.

Durch das Grafikprogramm Physiotools ${ }^{\circledR}$ (PhysioTools, 33100 Tampere, Finnland) wurden sowohl für die Therapeuten, als auch für die Patienten Übungsbögen erstellt. Damit konnten die Übungen, welche mit den Patienten durchge- 
Tab. 2 Verteilung der Diagnosen und Zuordnung zu den jeweiligen Gruppen

\begin{tabular}{lllll}
\hline & Gruppe A & Gruppe B & Gruppe C & Summe \\
\hline 1. Op nach Bandscheibenvorfall & 20 & 28 & 12 & 60 \\
\hline 2. Spondylodese & 4 & 17 & 11 & 32 \\
\hline 3. Langstreckige Fusion & 0 & 2 & 8 & 10 \\
\hline 4. Andere LWS-Op & 4 & 7 & 11 & 22 \\
\hline 5. Summe & 28 & 54 & 42 & 124 \\
\hline Op Operationn. & & & &
\end{tabular}

Tab. 3 t-Test für die Variablen Zeitpunkt t und ODI (max. 50 Punkte)

\begin{tabular}{|c|c|c|c|c|c|c|c|}
\hline Zeitpunkt t & $\mathrm{n}$ & $\begin{array}{l}\text { MW des } \\
\text { ODI abs. }\end{array}$ & $\begin{array}{l}\text { MW des } \\
\text { ODI (\%) }\end{array}$ & SD & $\begin{array}{l}\text { Standard- } \\
\text { fehler }\end{array}$ & $\begin{array}{l}\text { Minimum } \\
\text { des ODI }\end{array}$ & $\begin{array}{l}\text { Maximum } \\
\text { des ODI }\end{array}$ \\
\hline 1 & 124 & 21,1 & 0,4211 & 0,2084 & 0,0187 & 0 & 0,8400 \\
\hline 2 & 124 & 14.3 & 0,2854 & 0,2128 & 0,0191 & 0 & 0,8400 \\
\hline Diff (1-2) & & & 0,1356 & 0,2106 & 0,0267 & & \\
\hline
\end{tabular}

Tab. 4 t-Test für die Variablen Zeitpunkt t und 95\%-KI (max. 50 Punkte)

\begin{tabular}{|lllllll}
\hline Zeitpunkt t & MW & \multicolumn{2}{l}{$95 \%-K I$ des MW } & SD & \multicolumn{2}{l}{ 95\%-KI der SD } \\
\hline 1 & 0,4211 & 0,3840 & 0,4581 & 0,2084 & 0,1853 & 0,2381 \\
\hline 2 & 0,2854 & 0,2476 & 0,3233 & 0,2128 & 0,1892 & 0,2431 \\
\hline \multicolumn{7}{l}{ MW Mittelwert, SD Standardabweichung, KI Konfidenzintervall. } \\
\multicolumn{7}{l}{}
\end{tabular}

Tab. 5 t-Test für Methoden (max. 50 Punkte)

\begin{tabular}{lllll} 
Methode & Varianzen & Freiheitsgrade & t-Wert & Pr $>$ [t] \\
\hline Pooled & Gleich & 246,00 & 5,07 & $<0,0001$ \\
\hline Satterthwaite & Ungleich & 245,89 & 5,07 & $<0,0001$ \\
\hline
\end{tabular}

führt wurden, für Patient und Therapeut visualisiert werden, um somit eine möglichst hohe Koinzidenz der durchgeführten Übungen in allen vier Rehabilitationseinrichtungen zu gewährleisten.

Folgende Hypothesen wurden aufgestellt und statistisch überprüft.

- Die Werte für die verschiedenen Skalen sind nach der Reha signifikant besser als vor der Reha.

- Die Werte haben sich sowohl bei Frauen, als auch bei Männern verbessert.

- Die Werte innerhalb der Belastungsgruppe sind nach der Reha besser als vor der Reha.

In den Fragebögen wurden zur Überprüfung der Hypothesen u. a. die Variablen: Geschlecht, ODI, VAS, Art der Operation und die Reha-Gruppe erhoben. Die Überprüfung fand mittels t-Tests und Korrelationsanalysen statt. Da fehlende Werte in den zwei Zeitpunkten die Ergebnisse verzerren könnten, wurden die Tabellen für die Tests ODI, SF-12 und VAS von feh- lenden Werten für die Personen bereinigt. Dies vermeidet, dass die Ergebnisse der tTests durch einen systematischen Fehler verzerrt sind. Daher ergeben sich bei der Auswertung differente Patientenzahlen in den einzelnen untersuchten Auswertungssegmenten. Das Signifikanzniveau wurde $\alpha$ auf $5 \%$ festgelegt. Im Einzelnen erfolgte eine Auswertung getrennt nach Geschlechtern und Gruppen.

\section{Ergebnisse}

Entsprechend der Auswertung getrennt nach Geschlechtern und Gruppen konnte kein geschlechtsspezifischer Unterschied detektiert werden. In allen Gruppen zeigte sich eine signifikante Verbesserung in den drei Messinstrumenten ODI, SF-12 und VAS. Die Hypothese 1 „Die Werte für die verschiedenen Skalen sind nach der Reha signifikant besser als vor der Reha" konnte bestätigt werden.

Der durchschnittliche ODI vor der Reha (Zeitpunkt t1) lag bei 21,1 (0,4211), nach der Reha fand sich ein Wert von 14,3
(0,2850; t2). Die $95 \%$-Konfidenzintervalle der Mittelwerte überschneiden sich nicht, somit ist der beobachtete Unterschied signifikant (• Tab. 3, • Tab. 4, • Tab. 5, - Abb. 1).

Das gleiche Bild zeigt sich auch für die Auswertung der Werte der VAS. Der Mittelwert im Zeitpunkt t1 ist 5,04 (95\%-KI:4,55; 5,52) und zum Zeitpunkt t2 3,32 (95\%-KI:2,90; 3,74). Der Unterschied zu den Zeitpunkten $\mathrm{t} 1$ und $\mathrm{t} 2$ ist ebenfalls signifikant (• Tab. 6).

Auch bei der Auswertung des SF12-Fragebogens zeigen sich signifikante Unterschiede bei der körperlichen Skala zwischen Zeitpunkt t 1 und $\mathrm{t}$ 2, lediglich im Bereich der psychologischen Skala des SF-12 fand sich ein nicht signifikantes Ergebnis.

Um die Richtung dieses Unterschieds zu ermitteln wurden nun die Korrelation zwischen dem Zeitpunkt und dem Schmerzempfinden errechnet. Für den ODI besteht der Korrelationswert -0,31, für die VAS - 0,35. Daraus wird ein Unterschied in negativer Richtung geschlossen. Die subjektive Wahrnehmung des Gesundheitszustands verbesserte sich deutlich. Die Werte haben sich sowohl bei Frauen, als auch bei Männern signifikant verbessert

\section{Geschlecht und ODI}

Zum Zeitpunkt t1 haben 45 Männer einen errechneten ODI-Mittelwert von 0,4008 und zu Zeitpunkt t2 von 0,2243 (95\%$\mathrm{KI}=0,3410-0,4605$ und 0,2243-0,3495). Die Mittelwerte der 79 Frauen waren zum Zeitpunkt t1 0,4326 und 0,2846 zum Zeitpunkt t2 (95\%-KI = 0,3847-0,4806) und $(0,2361-0,3331)$. Die Unterschiede sind sowohl bei den Männern (0,0095) als auch bei den Frauen $(<0,0001)$ signifikant und in negativer Richtung, werden also niedriger, d. h. der Schmerz wurde geringer. Der Korrelationskoeffizient war -0,2723 bei den Männern und -0,32694 bei den Frauen.

\section{Geschlecht und VAS}

Für den VAS zeigt sich das gleiche Bild, wie für den ODI. Bei beiden Geschlechtern verändern sich die Werte für den Schmerz signifikant in negative Richtung 


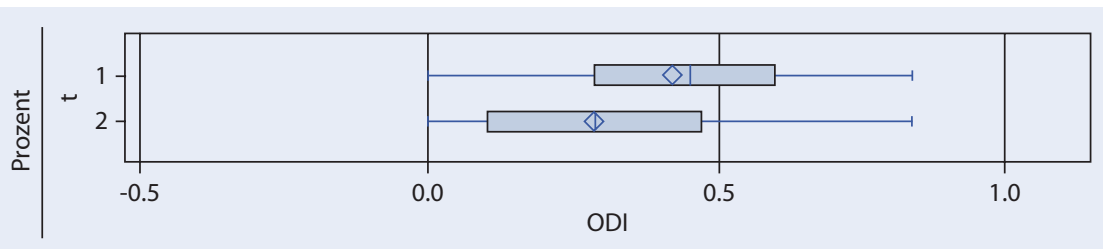

Abb. 1 Boxplot: ODI zu den Zeitpunkten eins und zwei (1,0=50 Punkte)

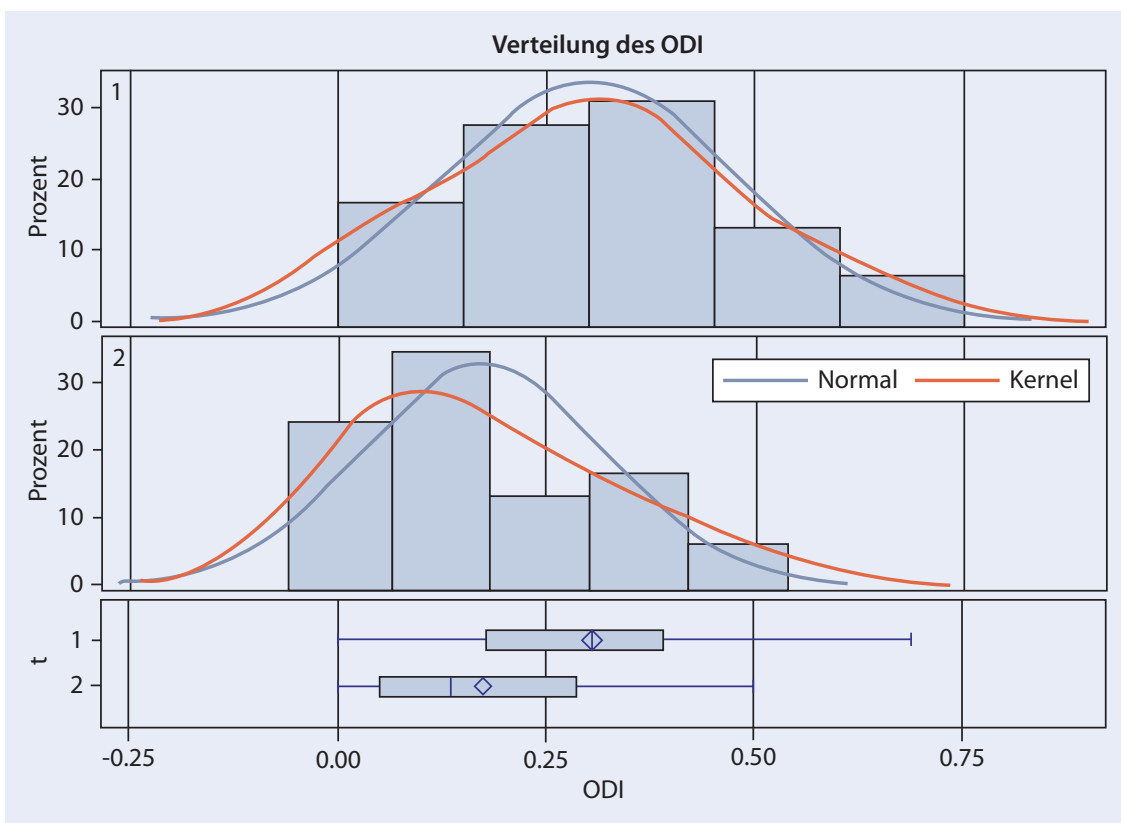

Abb. 2 \ Verteilung des ODI zu den zwei Zeitpunkten für Übungsgruppe 1 (1,0=50 Punkte)

(- Tab. 6). Die Werte innerhalb der Belastungsgruppen sind nach der Reha signifikant besser als vor der Reha.

\section{Belastungsgruppe A und ODI}

Es gab 28 Patienten, die in der anspruchsvollsten Behandlungsgruppe waren und den ODI-Fragebogen zu beiden Zeitpunkten ausgefüllt haben. Im Mittel hatten sie zu Zeitpunkt $\mathrm{t} 1$ einen ODI von $15,2(0,3038 ; 95 \%-\mathrm{KI}=0,2351-0,3726)$ und zu Zeitpunkt t2 einen ODI von 8,69 (0,1738; $95 \%-\mathrm{KI}=0,1171-0,2306)$. In der Belastungsgruppe A ist der Unterschied zwischen den Zeitpunkten $\mathrm{t} 1$ und $\mathrm{t} 2$ signifikant besser (• Tab. 8, • Abb. 2)

\section{Belastungsgruppe B und ODI}

Es gab 53 Patienten, die in der „mittleren" Belastungsgruppe waren und den ODI-Fragebogen zu beiden Zeitpunkten ausgefüllt haben. Im Mittel hatten sie zu Zeitpunkt $t 1$ einen ODI von 20,9 (0,4172;
$95 \%-\mathrm{KI}=0,3587-0,4756)$ und zu Zeitpunkt $\mathrm{t} 2$ einen ODI von $15,5(0,3102$; $95 \%-\mathrm{KI}=0,2471-0,3732$ ).

\section{Belastungsgruppe C und ODI}

Es gab 80 Patienten, die von den Operateuren in die Gruppe C mit den größten Einschränkungen eingeteilt waren und den ODI Fragebogen zu beiden Zeitpunkten ausgefüllt haben. Im Mittel hatten sie zu Zeitpunkt t1 einen ODI von 25,5 (0,5099; $95 \%-K I=0,4495-0,5704)$ und zu Zeitpunkt t 2 einen ODI von 16,6 $(0,3322 ; 95 \%-\mathrm{KI}=0,2640-0,4004)$. Auch hier ist der Unterschied für den ODI zum Zeitpunkt $\mathrm{t} 1 \mathrm{zu}$ t2 wie auch in den anderen beiden Gruppen signifikant. Die These kann damit, auf den ODI bezogen, bestätigt werden (• Tab. 7, • Tab. 8).

\section{Belastungsgruppe A und VAS}

Es gab 24 Patienten, die in der anspruchsvollsten Belastungsgruppe waren und die
VAS zu beiden Zeitpunkten ausgefüllt haben. Im Mittel hatten sie zu Zeitpunkt t1 einen Wert von 3,83 (95\%-KI = 2,90$4,76)$ und zu Zeitpunkt t 2 einen Wert von 2,90 (95\%-KI = 1,59-2,81). Der Korrelationskoeffizient beträgt $-0,407$, der Effekt ist damit in negativer Richtung, was bedeutet, dass der VAS niedriger geworden ist.

\section{Belastungsgruppe B und VAS}

Es gab 44 Patienten, die in der „mittleren“ Belastungsgruppe waren und die VAS zu beiden Zeitpunkten ausgefüllt haben. Im Mittel hatten sie zu Zeitpunkt t1 einen Wert von 5,25 (95\%-KI=4,48-6,01) und zu Zeitpunkt t 2 einen Wert von 3,63 $(95 \%-\mathrm{KI}=2,91-4,35)$.

\section{Belastungsgruppe C und VAS}

Es gab 32 Patienten, die der Belastungsgruppe $\mathrm{C}$ zugeordnet waren und die VAS zu beiden Zeitpunkten ausgefüllt haben. Im Mittel hatten sie zu Zeitpunkt t1 einen Wert von 5,65 (95\%-KI = 4,76$6,55)$ und zu Zeitpunkt $\mathrm{t} 2$ einen Wert von $3,71(95 \%-\mathrm{KI}=2,95-4,48)$. Auch hier ist der Unterschied für den VAS zum Zeitpunkt $\mathrm{t} 2 \mathrm{zu} \mathrm{t} 2$, wie auch in den anderen beiden Gruppen, signifikant. Die These kann damit auf den VAS bezogen bestätigt werden (• Tab. 8).

Letztlich können alle drei aufgestellten Hypothesen bestätigt werden. Den Patienten in allen drei Belastungsgruppen, Frauen wie Männer, ging es insgesamt zu Zeitpunkt t 2 bezogen auf Beeinträchtigungen durch Rückenschmerzen und die gesundheitsbezogene Lebensqualität signifikant besser als zu Zeitpunkt $t 1$.

\section{Diskussion}

Die Evidenz postoperativer Physiotherapiekonzepte nach Spondylodesen und anderen Eingriffen im Bereich der Lendenwirbelsäule (LWS) ist sehr klein, es existieren fast keine Untersuchungen, wobei auch das physiotherapeutische Management nach lumbalen Spondylodesen unklar und intransparent bleibt [3, 15, 17]. Nach Mikrodiskektomien und Dekompression von Spinalkanalstenosen scheint ein intensiviertes physiothe- 
Tab. 6 Geschlecht und VAS

\begin{tabular}{|llllll}
\hline $\begin{array}{l}\text { Ge- } \\
\text { schlecht }\end{array}$ & N & $\begin{array}{l}\text { MW zum Zeitpunkt 1 } \\
(\mathbf{9 5 \% - K I )}\end{array}$ & $\begin{array}{l}\text { MW zum Zeitpunkt } \\
\mathbf{2 ( 9 5 \% - K I )}\end{array}$ & $\operatorname{Pr}>|\mathbf{t}|$ & $\begin{array}{l}\text { Korrelationskoef- } \\
\text { fizient (Richtung) }\end{array}$ \\
\hline Männer & 40 & $4,8000(4,0467-5,5533)$ & $2,9750(2,3334-3,6166)$ & 0,0004 & $-0,3891$ \\
\hline Frauen & 63 & $5,1905(4,5446-5,8363)$ & $3,5397(2,9750-4,1043)$ & 0,0002 & $-0,3265$ \\
\hline \multicolumn{7}{l}{ MW Mittelwert, SD Standardabweichung, KI Konfidenzintervall. } \\
\end{tabular}

Tab. 7 Übungsgruppe und ODI (1,0=50 Punkte)

\begin{tabular}{|llllll}
\hline $\begin{array}{l}\text { Behand- } \\
\text { lungsgrup- } \\
\text { pe und ODI }\end{array}$ & $\mathbf{n}$ & $\begin{array}{l}\text { MW zum Zeitpunkt 1 } \\
(\mathbf{9 5} \%-K I)\end{array}$ & $\begin{array}{l}\text { MW zum Zeitpunkt 2 } \\
(95 \%-K I)\end{array}$ & $\operatorname{Pr}>|\mathbf{t}|$ & $\begin{array}{l}\text { Korrelations- } \\
\text { koeffizient } \\
\text { (Richtung) }\end{array}$ \\
\hline 1 & 28 & $0,3038(0,2351-0,3726)$ & $0,1738(0,1171-0,2306)$ & 0,0042 & $-0,3780$ \\
\hline 2 & 53 & $0,4172(0,3587-0,4756)$ & $0,3102(0,2471-0,3732)$ & 0,0141 & $-0,23777$ \\
\hline 3 & 40 & $0,5099(0,4495-0,5704)$ & $0,3322(0,2640-0,4004)$ & 0,0002 & $-0,40783$ \\
\hline MW Mittelwert KI Konfidenzintervall & & &
\end{tabular}

Tab. 8 Übungsgruppe und VAS

\begin{tabular}{|lllllll}
\hline $\begin{array}{l}\text { Behand- } \\
\text { lungsgrup- } \\
\text { pe und VAS }\end{array}$ & $\mathbf{n}$ & $\begin{array}{l}\text { MW zum Zeitpunkt 1 } \\
(\mathbf{9 5} \%-\mathrm{KI})\end{array}$ & $\begin{array}{l}\text { MW zum Zeitpunkt 2 } \\
\mathbf{9 5 \% - K I )}\end{array}$ & $\mathbf{P r}>|\mathbf{t}|$ & $\begin{array}{l}\text { Korrelations- } \\
\text { koeffizient } \\
\text { (Richtung) }\end{array}$ \\
\hline 1 & 24 & $3,8333(2,9043-4,7624)$ & $2,9043(1,5986-2,8181)$ & 0,0040 & $-0,40733$ \\
\hline 2 & 44 & $5,2500(4,4878-6,0122)$ & $3,6364(2,9148-4,3579)$ & 0,0026 & $-0,23777$ \\
\hline 3 & 32 & $5,6563(4,7608-6,5517)$ & $3,7188(2,9568-4,4807)$ & 0,0001 & $-0,39256$ \\
\hline \multicolumn{4}{l}{ MW Mittelwert, KI Konfidenzintervall. } & & & \\
\hline
\end{tabular}

rapeutisches Behandlungskonzept von Vorteil $[6,12]$. Primäres Ziel der vorliegenden Studie war die Analyse einer neu strukturierten Rehabilitation in der subakuten Behandlungsphase nach Operationen an der LWS. Strukturierte Nachbehandlungskonzepte fehlen, was in einer Untersuchung in England, die auch auf Deutschland übertragbar ist, sehr gut herausgearbeitet wurde [16].

In der vorliegenden Studie wurden die untersuchten Patienten nach Beendigung der Akutbehandlungsphase einer ambulanten oder stationären Rehabilitation im Rahmen einer Anschlussheilbehandlung zugeführt, in der Regel ab der 3. postoperativen Woche. Ein so frühzeitiger Beginn der Rehabilitation ist in der Literatur kaum beschrieben. Die wenigen Publikationen, die sich mit dieser Thematik befassen, unterscheiden meist einen Beginn nach 6 Wochen und 12 Wochen nach lumbaler Fusion [14] und zeigen keinen Unterschied im Outcome.

Das Patientenkollektiv ist zwar sehr inhomogen, die Anforderungen an eine Rehabilitation unterscheiden sich aber heute nicht nach der Ausgangsdiagnose, sondern am Zustand des Patienten und der erreichten Stabilität der Wirbelsäulenope- ration. Insofern ist es nur konsequent, die Einteilung durch den jeweiligen Operateur vornehmen zu lassen. Standards und Schemata erleichtern die konsequente gleichartige Nachbehandlung in definierten Behandlungsgruppen (• Tab. 1).

Die strukturierte Rehabilitation kann die Funktionsfähigkeit im Alltag nach der subakuten Behandlungsphase erhöhen. Da sowohl der ODI als auch die körperlichen Items des SF-12 alltägliche Aktivitäten abbilden, konnte durch die aktuelle Studie belegt werden, dass die strukturierte Rehabilitation in der Lage ist, die Funktionsfähigkeit im Alltag nach der subakuten Behandlungsphase zu erhöhen [9-11]. Die höhere prädiktive Validität der Funktionsfähigkeit lässt sich theoriekonform durch die starke Vorhersagekraft des ODI erklären, der die Beeinträchtigungen durch Rückenschmerzen erfasst. Parallel war eine ebenfalls signifikante Verringerung des Schmerzes, gemessen mit der VAS, zu verzeichnen.

Es konnte gezeigt werden, dass während der Durchführung der rehabilitativen Maßnahmen keine Notwendigkeit einer Reoperation herbeigeführt wurde. Bezüglich der Bewertung des Rehabilitationserfolgs muss eingeschränkt festgestellt werden, dass keine Kontrollgruppe parallel geführt wurde. Auch wurden nur Patienten aufgenommen, die Reha-fähig waren (sich eigenständig versorgen und selbständig an verordneten Therapie teilnehmen konnten), was die Ergebnisse möglicherweise positiv beeinflusst hat.

Perspektivisch könnte die strukturierte Rehabilitation der subakuten Behandlungsphase aufgrund der positiven Aspekte flächendeckend in die orthopädische Rehabilitation implementiert werden. Außerdem ist eine Langzeitevaluation dieser neu strukturierten Rehabilitation geplant. Voraussetzung für Langzeitbeobachtung sowie für die Bestimmung der Rehabilitationseffekte ist eine empirische Überprüfung der Änderungssensitivität, wodurch sich Veränderungen über die Zeit abbilden lassen [5].

Einschränkend ist jedoch anzumerken, dass es sich aufgrund der Implementation im klinischen Alltag zwar um ein prospektiv, jedoch selektiv gewonnenes $\mathrm{Pa}$ tientenkollektiv handelt. Ebenfalls limitierend ist die fehlende Analyse in Abhängigkeit zu soziodemografischen und krankheitsbezogenen Merkmalen der Rehabilitanden.

\section{Fazit für die Praxis}
- Das neu strukturierte entwickelte Re- habilitationskonzept mit drei Belas- tungsgruppen in der subakuten Be- handlungsphase nach lumbalen Wir- belsäulenoperationen unabhängig von der Diagnose und dem gewähl- ten Therapieverfahren ist ein neuer Ansatz und konnte einen positiven Ef- fekt auf Lebensqualität und Rücken- schmerz zeigen.
- Es bedarf jedoch weiterer struktu- rierter Rehabilitationsforschung, um einen Langzeiteffekt und sozioökono- mische Erkenntnisse zu gewinnen.

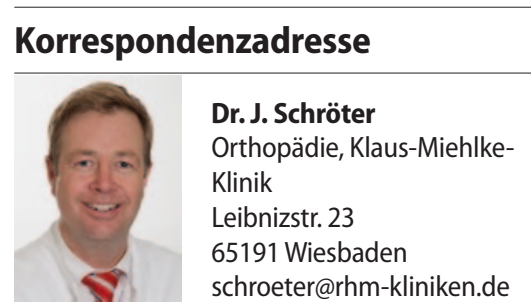


Danksagung. Diese Studie wurde für Patienten in der orthopädischen Rehabilitation nach LWS-Operationen im Rahmen mehrerer Konsensuskonferenzen als „Spinal Surgery Rehabilition Study“ durchgeführt.

\section{Einhaltung ethischer Richtlinien}

Interessenskonflikt. J Schröter, M. Lechterbeck F. Hartmann und E. Gercek geben an, dass kein Interessenkonflikt besteht

\begin{abstract}
Alle beschriebenen Untersuchungen am Menschen wurden mit Zustimmung der zuständigen Ethik-Kommission, im Einklang mit nationalem Recht sowie gemäß der Deklaration von Helsinki von 1975 (in der aktuellen, überarbeiteten Fassung) durchgeführt. Von allen beteiligten Patienten liegt eine Einverständniserklärung vor.
\end{abstract}

Open Access. Dieser Artikel unterliegt den Bedingungen der Creative Commons Attribution License. Dadurch sind die Nutzung, Verteilung und Reproduktion erlaubt, sofern der/die Originalautor/en und die Quelle angegeben sind.

\section{Literatur}

1. Fairbank JC, Pynsent PB (2000) The Oswestry disability index. Spine 25:2940-2952 (discussion 2952)

2. Frey C, Zwingmann C, Bocker M et al (2011) Adaptive testing in rehabilitation: a way to economic assessment of patients' attributes]. Rehabilitation 50:195-203
3. Gibson JN, Waddell G (2005) Surgery for degenerative lumbar spondylosis. Cochrane Database Syst $\operatorname{Rev}(2): C D 001352$ (Review. Update in: Cochrane Database Syst Rev (4):CD001352)

4. Grant S, Aitchison T, Henderson E et al (1999) A comparison of the reproducibility and the sensitivity to change of visual analogue scales, Borg scales, and Likert scales in normal subjects during submaximal exercise. Chest 116:1208-1217

5. Igl W, Zwingmann C, Faller H (2005) Sensitivity to change. Rehabilitation 44:100-106

6. Kohlmann T, Raspe H (1996) Hannover functional questionnaire in ambulatory diagnosis of functional disability caused by backache. Rehabilitation 35:I-VIII

7. Kulig K, Beneck GJ, Selkowitz DM et al (2009) An intensive, progressive exercise program reduces disability and improves functional performance in patients after single-level lumbar microdiskectomy. Phys Ther 89:1145-1157

8. Luo X, George ML, Kakouras I et al (2003) Reliability, validity, and responsiveness of the short form 12-item survey (SF-12) in patients with back pain. Spine 28:1739-1745

9. Mannion AF, Junge A, Fairbank JC et al (2006) Development of a German version of the Oswestry Disability Index. Part 1: cross-cultural adaptation, reliability, and validity. Eur Spine J 15:55-65

10. Mannion AF, Junge A, Grob D et al (2006) Development of a German version of the Oswestry Disability Index. Part 2: sensitivity to change after spinal surgery. Eur Spine J 15:66-73
11. Mayo NE, Poissant $L$, Ahmed S et al (2004) Incorporating the International Classification of Functioning, Disability, and Health (ICF) into an electronic health record to create indicators of function: proof of concept using the SF-12. J Am Med Inform Assoc 11:514-522

12. McGregor AH, Probyn $K$, Cro S, Doré CJ, Burton AK, Balagué F, Pincus T, Fairbank J (2013) Rehabilitation following surgery for lumbar spinal stenosis. Cochrane Database Syst Rev (12):CD009644. doi:10.1002/14651858.CD009644. (pub2.Review)

13. Morfeld M, Kirchberger I, Bullinger M (2011) SF36 Fragebogen zum Gesundheitszustand: Deutsche Version des Short Form-36 Health Survey 2., ergänzte und überarbeitete Auflage. Göttingen, Hogrefe

14. Oestergaard LG, Christensen FB, Nielsen CV et al (2013) Early versus late initiation of rehabilitation after lumbar spinal fusion: economic evaluation alongside a randomized controlled trial. Spine 38:1979-1985

15. Rushton A, Eveleigh G, Petherick EJ et al (2012) Physiotherapy rehabilitation following lumbar spinal fusion: a systematic review and meta-analysis of randomised controlled trials. BMJ Open 2

16. Rushton A, Heneghan N, Heap A et al (2014) Survey of current physiotherapy practice for patients undergoing lumbar spinal fusion in the UK. Spine(Phila Pa 1976) (Epub ahead of print)

17. Theiler R, Schmid C, Risler R et al (2007) Postoperative physiotherapy in acute care-when, what and how much? Orthopade 36:552, 554-559

\section{Springer Medizin}

\section{Neu: Aktuelle Operationstechniken im Video Reichen Sie Ihren Beitrag für Der Orthopäde jetzt ein!}

Im Mittelpunkt des Beitrags steht Ihr Video zu aktuellen Operationstechniken. Es werden bewährte operative Verfahren dargestellt. Neben der Vermittlung aller wichtigen prä-, intraund postoperativen Details sowie den Hinweisen auf mögliche

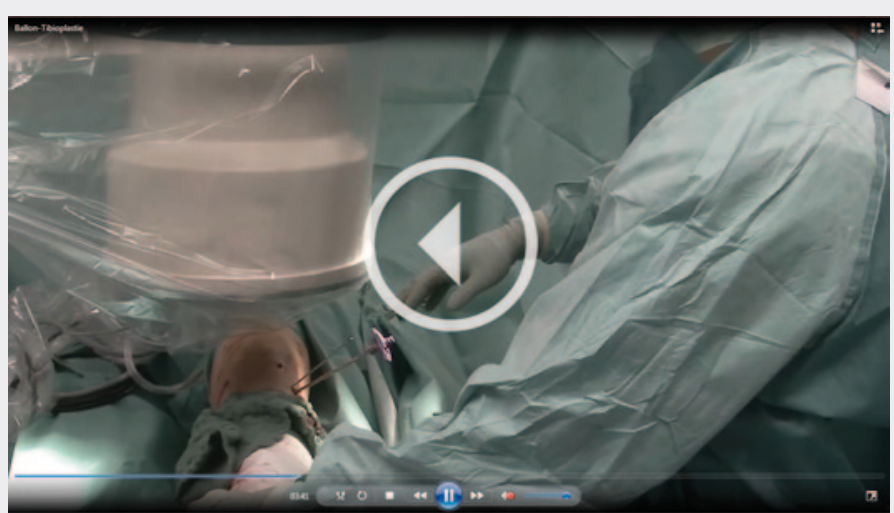

Alle Operationsschritte werden übersichtlich im Detail von Experten erklärt. Die neue Videoreihe finden Sie auf springermedizin.de
Fehler und Gefahren liegt der Schwerpunkt auf einer genauen Darstellung der jeweiligen Operationsschritte im Video. Nutzen Sie das Medium des Videos um komplizierte Vorgehensweisen leicht verständlich zu präsentieren. Bitte reichen Sie zudem einen kurzen begleitenden Beitrag ein, der die wesentlichen Punkte des Videos zusammenfasst. Das Copyright des Videos bleibt beim Autor.

\section{Weitere Informationenzur Einreichung sowie einen detaillierten Autorenleitfaden finden Sie auf www.DerOrthopaede.de sowie editorialmanager.com/deor/}

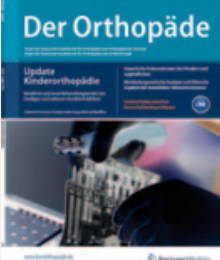

